\title{
The Three Pillars of Virtual Reality? Investigating the Roles of Immersion, Presence, and Interactivity
}

\author{
Joschka Mütterlein \\ LMU Munich \\ muetterlein@bwl.lmu.de
}

\begin{abstract}
Virtual reality (VR) technologies enable a new media consumption experience. Although VR's origins trace back at least to the 1960s, it is still unclear how $V R$ 's postulated key features immersion, presence, and interactivity contribute to that experience. Furthermore, it is unclear whether flow as a construct closely related to immersion offers explanatory power in investigating $V R$. On the basis of a quantitative survey in a VR center with 294 participants, I analyze the interplay of the key features and exemplify their influence in a VR context by relating them to satisfaction with the VR experience. Using a flowbased conceptualization of immersion, I find that presence as well as interactivity contribute to immersion. In addition, interactivity contributes to presence. Furthermore, my results show that immersion influences satisfaction with a VR experience, indicating that a flow-based conceptualization of immersion is a suitable predictor in VR contexts.
\end{abstract}

\section{Introduction}

In the last centuries, new technologies have enabled innovative products that have greatly changed the way media are consumed, e.g., photo, film, and video games. Virtual reality (VR) is the next technology that might have such disruptive potential. It can be defined as "the sum of the hardware and software systems that seek to perfect an all-inclusive, immersive, sensory illusion of being present in another environment" [8, p. 63].

VR possesses unique characteristics that stem from users being enveloped by and conducting activities in another environment while consuming VR content [49]. These characteristics are frequently described as the three key features immersion, presence, and interactivity [e.g., 9; 46; 53]. Especially immersion and presence are said to potentially reach higher levels in
VR than in previous media forms. However, the relation of the three characteristics to each other is unclear, as the majority of previous research covers them just partly [e.g., 27] or does not clearly distinguish between presence and immersion [33]. Moreover, empirical evidence on their joint influence on consumers' VR experience is scarce and suffers from different views on measurement instruments [47], especially when it comes to presence and immersion. In addition, research has recently questioned the choice of immersion as a key characteristic, suggesting that flow might be a closely related construct that offers more explanatory power in investigating VR [36].

Considering that immersion/flow, presence, and interactivity seem to be crucial in understanding VR, I explore their relation to each other and exemplify their influence in VR contexts by relating them to consumers' satisfaction with a VR experience. I focus on hedonic VR experiences, because these drive VR diffusion [51], and head-mounted VR devices, because these are most relevant for the market [4]. Against this backdrop, my research questions are:

(RQ1) How are immersion/flow, presence, and interactivity related?

(RQ2) How do immersion/flow, presence, and interactivity influence satisfaction with a VR experience?

Answering these research questions is highly relevant for theory and practice, because immersion/flow, presence, and interactivity each seem to influence users' perception of VR [e.g., 5; 23], but cannot occur isolated from another in a VR experience - their interplay is important. This study contributes to research by deepening understanding of this interplay and its effects in a VR context, by offering a flowbased approach to distinguishing presence and immersion, and by adding to first empirical evidence on the role of flow in VR consumption. Furthermore, it contributes to practice by exploring to what extent VR's characteristics drive consumers' satisfaction, providing insights for the design of VR experiences.

The remainder of this paper is structured as follows: First, I develop the background of this project 
by describing the current state of relevant VR and flow literature, before explaining hypotheses and the research model. Second, I outline the research design to test the hypotheses and present results. Third, I discuss results, derive implications for research and practice, and conclude with a short summary.

\section{Theoretical foundations}

\subsection{Virtual reality characteristics}

VR's technical origins trace back at least to the 1960 s, when a scientific version of an "ultimate display" was introduced [52]. In the following decades, the technology improved, which lead to many managerial and IS inquiries in the 1990s and 2000s $[43 ; 53]$.

During the same time, research has started to explore the "ultimate" nature of VR and addressed characteristics of VR, focusing strongly on presence or telepresence [49]. Presence describes "the subjective experience of being in one place or environment, even when one is physically situated in another" [55]. Telepresence refers to states of presence that are reached using a medium [49]. Until recently, the role of telepresence in VR contexts has been investigated from time to time [e.g., 23], but results are mixed [27].

Connections between presence and immersion were suggested early [e.g., 49; 55], but insights are limited due to mostly conceptual approaches, restricted VR capabilities of the $1990 \mathrm{~s}$ and $2000 \mathrm{~s}$, and different views on the definition and measurement of factors [e.g., 15; 48]. The latter becomes especially apparent in the discussion revolving around immersion. Some researchers view immersion as a state of mind, i.e. a subjective psychological experience, and define it, for example, as feeling caught up in and absorbed by the virtual world [33]. Other researchers see immersion as a technological capability of a VR system. This means that there are VR technologies that are more or less immersive, e.g., by using more or less sensors or having a larger or smaller field of view [47]. Following this line of argument, immersion would be assessable objectively using a VR system's technological capabilities rather than measuring consumers' subjective experience. However, this would also mean that different types of VR content that make use of the same VR technology, e.g., a large-budget racing game and a small-budget quiz game, do not differ in their level of immersion. From a behavioral research's standpoint, such an interpretation seems too simplified. Thus, I follow the view of immersion as a psychological experience that is certainly based on and restricted by the technological capabilities of a system, but has to be measured on a subjective level.

This view is also supported by insights from psychology on the nature of flow, which relates to "a state of optimal experience where one is completely absorbed and immersed in an activity" [38]. Flow is a subjective experience and usually described using different dimensions, many of them relating to feelings that occur in states of immersion, such as temporal dissociation [1] or merging of action and awareness [11]. Consequently, research has recently suggested focusing on flow to potentially be better able to assess immersion [36]. This seems all the more important as the role of flow in VR has been little explored so far [37], despite its large similarity to immersion and important role in other IS research streams [e.g., 1; 7]. As findings on the role of immersion are mixed depending on definition [33], measurement [47], and context [30], a flow-based conceptualization of immersion might indeed offer more explanatory power.

Aforementioned conflicting thoughts on the subjective vs. objective measurement of VRs' key features can also be applied to interactivity, i.e. "the degree to which users of a medium can influence the form or content of the mediated environment" [49]. Following the arguments made above, I also regard interactivity as a psychological state of mind, i.e. I focus on perceived interactivity. This is because a certain influence on the form or content of an environment might be viewed as more or less interactive depending on the individual and, e.g., her experience with VR. Research on the psychological dimensions of perceptions of interactivity supports such an interpretation [34]. Similar to telepresence and immersion, the importance of interactivity for VR was also highlighted early $[49 ; 55]$ and confirmed in some contexts recently [e.g., 5].

I exemplify the influence of VR's three key features using satisfaction as a dependent variable. Satisfaction is an individuals' feeling about or attitude towards a product or service that emerges as a result of an assessment of actual first-hand experience with it [6]. It can be captured as a positive feeling, i.e. satisfaction, or as an indifferent or negative feeling, i.e. dissatisfaction [2]. Research has found that the satisfaction level has large impact on consumers' intention to use a product or service again [6;40], which indicates that satisfaction can also be a key variable for VR diffusion and long-term market success. Yet, despite its potential importance, in a VR context it has only been investigated regarding satisfaction with therapy success [45]. Thus, insights are hardly transferable to satisfaction with overall VR use. 


\subsection{Hypotheses and research model}

My research model covers telepresence, a flowbased conceptualization of immersion, and interactivity as VR's key characteristics as well as satisfaction as a dependent variable. The relationships of telepresence, immersion, and interactivity are largely based on insights from previous research.

However, these insights do not always paint a clear picture. When immersion is seen as a technological capability of a VR system, it is likely that immersion leads to telepresence [47]. But when immersion is seen as a psychological experience, this relation becomes less clear. Early research in VR contexts postulates that the subjective feeling of immersion leads to telepresence [55], but this could depend on the structure of the factor used [54]. In addition, immersion and telepresence are often "so loosely defined as to be interchangeable" [33, p. 68]. This becomes apparent when comparing definitions: the essence of most definitions of telepresence is that a medium is used to create a subjective feeling that one is in another place $[49 ; 55]$. Definitions of immersion often relate to being caught up in another world [33]. To distinguish the two concepts more clearly, I follow the aforementioned definition of telepresence, but draw on flow literature to conceptualize immersion in VR as the subjective experience of feeling totally involved in and absorbed by the activities conducted in a place or environment, even when one is physically situated in another $[14 ; 38 ; 55]$. Such a definition allows distinguishing the subjective experience of being in another place in VR, i.e. telepresence, from the activities conducted there, i.e. immersion. Based on that, I argue that one must first feel being present in another place to become immersed in VR activities, because otherwise distractions from the real world prevent from reaching a state of immersion in the virtual world. This leads to the following hypothesis:

\section{$H_{1}$ : Telepresence has a direct and positive influence on immersion.}

The influence of interactivity on telepresence and immersion is less controversial. There is wide agreement that being able to interact with an environment, instead of just passively watching it, leads to feeling present in that environment [33; 49; 55]. Similarly, the majority of flow literature emphasizes that being able to control one's actions leads to higher states of immersion and flow $[14 ; 25$; 24]. I assume that this also applies to VR, which leads to the following hypotheses:

\section{$H_{2}$ : Interactivity has a direct and positive influence on telepresence.
$H_{3}$ : Interactivity has a direct and positive influence on immersion.

Regarding possible relations to satisfaction, research in non-VR contexts shows that presence [31] and immersion [17] can both increase satisfaction. Yet, it is not fully clear if these results are applicable to this project due to aforementioned issues with the distinction of presence and immersion. Research on flow has shown that flow influences satisfaction [24, 41] and even addiction [12] in different contexts. Although these effects do not necessarily have to be caused by the immersion-related dimensions of flow, but could also be due to other aspects of flow, overall evidence on a relationship of a flow-based conceptualization of immersion on satisfaction seems strong. Thus, I argue:

\section{$H_{4}$ : Immersion has a direct and positive influence on satisfaction.}

All four hypotheses are covered in the research model (see Figure 1). I assume that telepresence influences immersion, interactivity influences telepresence as well as immersion, and immersion influences consumers' satisfaction with a VR experience.

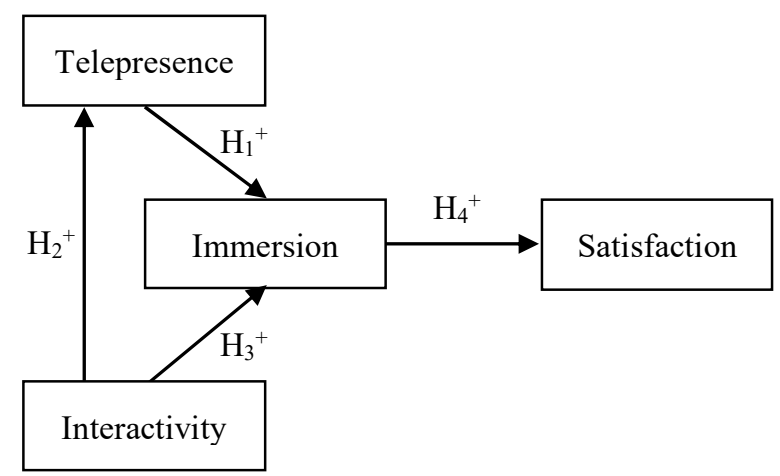

Figure 1. Research model

\section{Method}

To investigate my hypotheses, I have conducted a quantitative survey with a VR center as a business partner. This VR center offers visitors state of the art VR technology to consume VR content of their choice. On average, 30 minutes of VR consumption cost 10 Euros, but participants did not have to pay for the 
content consumed during the study. By collaborating with the VR center, I could perform this study in a real life setting using HTC Vive devices as VR equipment. Over the course of two weeks, 294 visitors of the center participated. Their gender distribution was nearly equal with a slight bias towards men, average age was 28 , and only roughly $40 \%$ were students. All in all, this indicates that my study is close to being representative for the main target group of hedonic VR content.

After coming to the center, participants needed to complete the first part of the survey asking them about demographics and other control variables such as income, monthly media spending, experience with VR, or possession of VR equipment. Then, participants were assigned to test one of two preselected VR contents ("The Body VR" or "VR The Diner Duo") to reduce possible bias in the data due to characteristics of one specific VR content. Both VR experiences lasted for 12 minutes, followed by the second part of the survey covering the research model. Testing VR content directly before answering questions about, e.g., flow enabled me to assess effects directly and not just from a distant point in time. Per participant, the whole procedure took about 50 minutes. I tested and adapted it before the actual study in two rounds of pretests with 13 IS researchers and participants without a scientific background. Following data collection, data were analyzed using SPSS for confirmatory factor analyses and SmartPLS for structural equation modeling (SEM) [44].

To ensure that all items were covered in accordance with my definitions, I carefully combined and adapted established scales from literature (see Table 1). I covered telepresence based on items from Animesh et al. [2], Nah et al. [37], and Nelson et al. [39]; immersion based on an immersion-focused version of Rheinberg's flow short scale, consisting of the two dimensions fluency and absorption that were modeled as a reflective second order construct [42]; interactivity based on items and definitions from Animesh et al. [2], Johnson et al. [26], and Steuer [49]; and satisfaction with items from Bhattacherjee [6]. To be able to test for common method bias [28], I also added political

Table 1. Constructs and results of factor analyses

\begin{tabular}{|c|c|c|c|c|c|}
\hline \multicolumn{2}{|c|}{ Construct } & Mean & $\begin{array}{l}\text { Std. } \\
\text { Dev. }\end{array}$ & $\begin{array}{r}\text { Factor } \\
\text { loading }\end{array}$ & $\begin{array}{r}\text { Cronbach's } \\
\text { Alpha }\end{array}$ \\
\hline \multicolumn{5}{|c|}{ Telepresence } & \multirow{5}{*}{0.790} \\
\hline TP1 & $\begin{array}{l}\text { The VR content created a new world for me, and this new } \\
\text { world suddenly disappeared when the VR content ended. }\end{array}$ & 4.83 & 1.709 & 0.836 & \\
\hline TP2 & $\begin{array}{l}\text { When the VR content ended, I felt as if I returned to the "real } \\
\text { world" after a journey. }\end{array}$ & 4.79 & 1.718 & 0.783 & \\
\hline TP3 & $\begin{array}{l}\text { I forgot about my immediate surroundings when I was using } \\
\text { the VR content. }\end{array}$ & 4.36 & 1.694 & 0.746 & \\
\hline TP4 & $\begin{array}{l}\text { The VR content seemed to be "somewhere I visited" rather } \\
\text { than "something I saw". }\end{array}$ & 4.83 & 1.697 & 0.766 & \\
\hline \multicolumn{5}{|c|}{ Immersion - Fluency } & \multirow{4}{*}{0.723} \\
\hline FL1 & I had no difficulty concentrating. & 5.42 & 1.501 & 0.797 & \\
\hline FL2 & My mind was completely clear. & 5.42 & 1.382 & 0.862 & \\
\hline FL3 & $\begin{array}{l}\text { The right thoughts and movements occurred of their own } \\
\text { accord. }\end{array}$ & 5.18 & 1.474 & 0.751 & \\
\hline \multicolumn{5}{|c|}{ Immersion - Absorption } & \multirow{4}{*}{0.890} \\
\hline AB1 & I didn't notice time passing. & 4.58 & 1.862 & 0.912 & \\
\hline AB2 & I was totally absorbed in what I was doing. & 5.04 & 1.719 & 0.914 & \\
\hline AB3 & I was completely lost in thought. & 3.77 & 1.848 & 0.891 & \\
\hline \multicolumn{5}{|c|}{ Interactivity } & \multirow{4}{*}{0.920} \\
\hline IT1 & The VR content allowed me to interact with the virtual world. & 3.86 & 2.149 & 0.918 & \\
\hline IT2 & $\begin{array}{l}\text { I had the feeling that I could influence the virtual world of the } \\
\text { VR content. }\end{array}$ & 3.01 & 2.046 & 0.933 & \\
\hline IT3 & The VR content was interactive. & 3.47 & 2.189 & 0.937 & \\
\hline \multicolumn{5}{|c|}{ Satisfaction } & \multirow{4}{*}{0.852} \\
\hline SA1 & Very dissatisfied-Very satisfied & 4.70 & 1.487 & 0.884 & \\
\hline SA2 & Very displeased-Very pleased & 4.91 & 1.311 & 0.911 & \\
\hline SA3 & Very frustrated-Very contented & 4.75 & 1.145 & 0.849 & \\
\hline
\end{tabular}


interest based on Francis \& Greer [18] as a marker variable to the survey. All questions were answered on Likert scales ranging from 1 - strongly disagree to 7 strongly agree. The only exception was satisfaction. Here, participants answered different questions regarding their feelings about their experience on a Likert scale from 1 to 7 with different wording (see Table 1). In addition, all items provided an "I don't know" option for participants that could not decide for one clear answer.

As the study was conducted in Germany, I translated all items from their original English wording to German in a systematic process. I translated the original item from English to German. Two other researchers translated the wording back to English independent from another. The original and translated English versions were compared by a fourth researcher, who then decided whether the translated items matched the meanings of the original items. In the rare case of both translations differing from their original, all four researchers discussed improvements of the translation and decided with full consent.

\section{Results}

During factor analysis, I assessed factor loadings [32] and reliability values [13], amongst others. All items had satisfactory factor loadings greater than 0.7 [32], except one item for satisfaction. Accordingly, it was excluded from further analyses. The remaining scales (see Table 1, only items and values shown after scale trimming) had reliable Cronbach's Alpha values above 0.7 [13], providing a sound foundation for SEM. I used a partial-least-squares (PLS) approach because it is better able to handle smaller sample sizes than covariance-based approaches and, thus, fits the data better $[10 ; 22]$. In addition, it has already proved to be suitable in similar contexts [23].

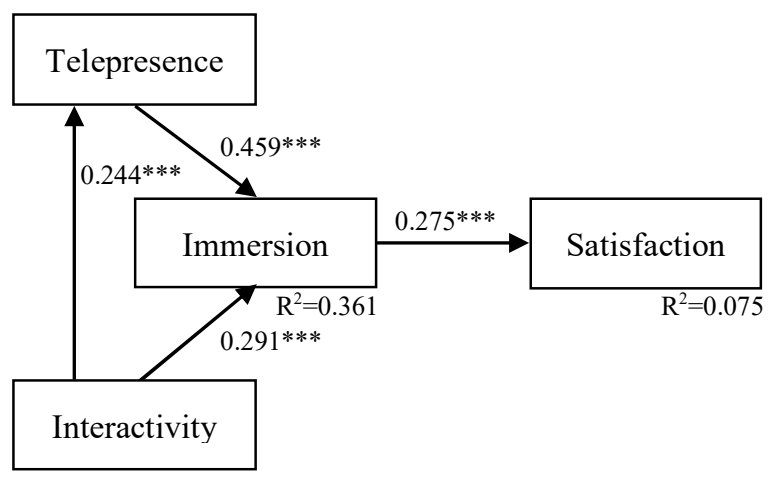

Figure 2. SEM results
Using PLS-SEM, I evaluated relationships of the model [29], significance of effects via bootstrapping [20], predictive relevance $[19 ; 50]$, convergent and discriminant validity $[10 ; 16]$, and variance inflation factors [21], amongst others. The tests for predictive relevance, validity, and non-collinearity were within their respective thresholds. Testing correlations of the marker variable with the model's variables did not yield any correlations, indicating that common method bias [28] is not a problem in my study.

Table 2. Hypotheses tests

\begin{tabular}{|l|r|}
\hline Hypothesis & Result \\
\hline $\begin{array}{l}\mathrm{H}_{1}: \text { Telepresence has a direct and } \\
\text { positive influence on immersion. }\end{array}$ & Supported \\
\hline $\begin{array}{l}\mathrm{H}_{2}: \text { Interactivity has a direct and } \\
\text { positive influence on telepresence. }\end{array}$ & Supported \\
\hline $\begin{array}{l}\mathrm{H}_{3}: \text { Interactivity has a direct and } \\
\text { positive influence on immersion. }\end{array}$ & Supported \\
\hline $\begin{array}{l}\mathrm{H}_{4}: \text { Immersion has a direct and } \\
\text { positive influence on satisfaction. }\end{array}$ & Supported \\
\hline
\end{tabular}

The results of relationship evaluation and significance testing are shown in Figure 2. All effects and hypotheses were supported as assumed (see Table 2) with high significance levels $(p<0.001)$. I found strong influence of telepresence and interactivity on immersion, their effects leading to an explained variance of $\mathrm{R}^{2}=0.361$. In addition, $\mathrm{I}$ found that interactivity influences telepresence. Finally, I found a highly significant effect of immersion on satisfaction, although the amount of variance explained is rather small $\left(\mathrm{R}^{2}=0.075\right)$.

\section{Discussion and implications}

My results demonstrate that the interplay of telepresence, immersion, and interactivity is important in analyzing and understanding VR experiences. Interactivity is an important foundation contributing to telepresence as well as immersion. Telepresence seems crucial for immersion. However, this relation depends heavily on a precise differentiation of telepresence and immersion, as well regarding definitions as measures. I have established such a differentiation using a definition of presence that focuses on feeling as being in another place [55], while my definition of immersion draws on flow literature and relates to feeling involved in and absorbed by the activities conducted in that place $[14 ; 38]$. In addition, I have carefully built scales based on relevant previous literature that closely resemble the focus of the 
definitions. This results in a relation of telepresence and immersion that is different from previous literature [e.g., 33; 47; 55], but identifies strong and significant explanations for the interplay of VR's key features.

In addition, this interplay has a significant effect on consumer's VR experience, as I exemplify with the effect of immersion on satisfaction. The rather low $\mathrm{R}^{2}$ indicates that there are additional factors explaining satisfaction with VR experiences, but identifying these and explaining satisfaction with VR experiences is not the scope of this paper. Instead, on the one hand findings underline that telepresence, immersion, and interactivity have to be considered together to comprehensively explore VR; on the other hand they indicate that a flow-based conceptualization of immersion is well suited as a predictor in VR contexts.

For research, these insights offer a solid foundation to further investigate VR. For example, the three key features can be used to increase explanatory power of models concerning VR acceptance and use. While there is already some research covering these topics and demonstrating a certain influence of telepresence or interactivity [e.g., 5; 23], VR's specifics have not yet been covered systematically.

A second avenue for further research is the distinction of telepresence and immersion. Discussions about subjective or objective measurement $[47 ; 55]$ as well as about unclear definitions [33] have accompanied VR research from its beginnings. This has led to vague specifications of constructs in empirical research [36], which makes it hard to transfer insights from previous research on presence and immersion to novel contexts, especially VR. The distinction proposed in this paper contributes to resolving such issues by limiting the presence construct to its core feature, i.e. the feeling of being present in another place. The immersion construct is bound to dimensions of flow that resemble immersion in VR, i.e. feeling totally involved in and absorbed by the activities conducted in this other place. My empirical study seems to strongly support the effect of telepresence on immersion that results from this distinction. Nevertheless, the underlying assumption of causality will benefit from further evidence. Additionally, the proposed definitions and the scales developed and confirmed in the course of this paper will benefit from critical examination, adaption, and use in other contexts.

Finally, a third avenue for research is based on the role of flow in VR examinations. So far, evidence on the role of flow is scarce [37], and this paper only considers selected, immersion-related aspects of flow. Still, my findings add to the emerging evidence for flow as an important part of a VR experience. However, flow covers other aspects as well [14], such as enjoyment, which might be necessary to explore VR in depth. Thus, the relation of other flow dimensions to the postulated three key features of VR needs inquiry, not only empirical, but also conceptual to distinguish all concepts clearly from another.

For practice, my findings provide guidance in producing VR content and improving VR technology. For example, features that enhance interactivity and make users feel present in VR seem beneficial to create a state of immersion. This indicates that media companies' current focus on producing passively viewed $360^{\circ}$ videos [35] might not be beneficial. Rather, investments in more interactive VR content seem advisable.

\section{Summary, limitations and conclusion}

This projects' aim was to explore how immersion, presence, and interactivity are related. In addition, I wanted to exemplify their influence by investigating satisfaction with a VR experience. Based on an examination of related literature, I first distinguished presence and immersion more clearly by limiting the focus of presence on feelings of being in another place and by conceptualizing immersion based on flow. Using a quantitative survey with 294 participants and relying on scales carefully put together to be in accordance with this distinction of presence and immersion, I found that interactivity influences telepresence while immersion and telepresence influence immersion. This flow-based conceptualization of immersion in turn influences satisfaction with the VR experience. These findings advance research by clarifying the interplay and effect of VR's postulated key features, by suggesting a clear and measurable distinction of presence and immersion, and by highlighting the potential of flow for VR research. For practitioners, insights on the influence of VR features can help in creating more appealing VR content and technologies.

I have already outlined some areas for further research based on my findings. In addition to these, areas for further research stem from the limitations of this work: For example, the role of the three key features needs to be confirmed in a non-hedonic context, as the focus of this work and its object of investigation is hedonic VR content. In addition, I have used advanced head-mounted VR devices to evaluate hypotheses. Although there is little doubt that the technology will improve even more in the future, making currently advanced systems the norm, it is not clear whether insights are applicable to systems at the lower end of the market. Furthermore, the surroundings of my study, i.e. the business partners' premises, might 
have influenced results [36]. Thus, investigating VRs' characteristics in other surroundings is necessary. Finally, investigating factors influencing satisfaction with a VR experience was beyond the scope of this paper, but could lead to a better understanding of mechanisms that drive the intention to continue using VR, which can be seen as a precondition for the technology's long-term market success [6]. Further research should address satisfaction with a VR experience in more depth and clarify how VR brings value to customers.

All in all, my project demonstrates that although the technology has been around and investigated for many years, it is still not fully clear what makes VR special from a behavioral perspective. Investigating the role of immersion, presence, and interactivity is arguably an important building block, but certainly not the end.

\section{References}

[1] R. Agarwal, and E. Karahanna, "Time Flies When You're Having Fun: Cognitive Absorption and Beliefs about Information Technology Usage", MIS Quarterly (24:4), 2000, pp. 665-694.

[2] R.E. Anderson, "Consumer Dissatisfaction: The Effect of Disconfirmed Expectancy on Perceived Product Performance", Journal of Marketing Research (10:1), 1973, pp. 38-44.

[3] A. Animesh, A. Pinsonneault, S.-B. Yang, and W. Oh, "An Odyssey into Virtual Worlds: Exploring the Impacts of Technological and Spatial Environments on Intention to Purchase Virtual Products", MIS Quarterly (35:3), 2011, pp. 789-810.

[4] C. Anthes, R.J. García-Hernández, M. Wiedemann, and D. Kranzlmüller, "State of the Art of Virtual Reality Technology", Proceedings of the IEEE Aerospace Conference, 2016, Big Sky, MT.

[5] J. Bailenson, K. Patel, A. Nielsen, R. Bajscy, S.-H. Jung, and G. Kurillo, "The Effect of Interactivity on Learning Physical Actions in Virtual Reality", Media Psychology (11:3), 2008, pp. 354-376.

[6] A. Bhattacherjee, "Understanding Information Systems Continuance: An Expectation-Confirmation Model", MIS Quarterly (25:3), 2001, pp. 351-370.

[7] A. Bilgihan, K. Nusair, F. Okumus, and C. Cobanoglu, "Applying Flow Theory to Booking Experiences: An Integrated Model in an Online Service Context", Information \& Management (52:6), 2015, pp. 668-678.

[8] F. Biocca, and B. Delaney, "Immersive Virtual Reality Technology", in Biocca, F., and M.R. Levy (eds.),
Communication in the Age of Virtual Reality, Lawrence Erlbaum, Hillsdale, NJ, 1995, pp. 57-124.

[9] G.C. Burdea, and P. Coiffet, Virtual Reality Technology, John Wiley \& Sons, Hoboken, NJ, 2003.

[10] W.W. Chin, "The Partial Least Squares Approach to Structural Equation Modeling", in Marcoulides G.A. (ed.), Modern Methods for Business Research, Lawrence Erlbaum, Mahwah, NJ, 1998, pp. 295-336.

[11] B. Choi, and Y. Baek, "Exploring Factors of Media Characteristic Influencing Flow in Learning Through Virtual Worlds", Computers \& Education (57:4), 2011, pp. 23822394.

[12] T.-J. Chou, and C.-C. Ting, "The Role of Flow Experience in Cyber-Game Addiction", Cyberpsychology \& Behavior (6:6), 2003, pp. 663-680.

[13] L. Cronbach, "Coefficient Alpha and the Internal Structure of Tests", Psychometrika (16:3), 1951, pp. $297-$ 334.

[14] Csikszentmihályi, M., Flow: The Psychology of Optimal Experience, Harper and Row, New York, NY, 1990.

[15] J.V. Draper, D.B. Kaber, and J.M. Usher, "Telepresence", Human Factors (40:3), 1998, pp. 354-375.

[16] C. Fornell, and D.F. Larcker, "Structural Equation Models with Unobservable Variables and Measurement Error: Algebra and Statistics", Journal of Marketing Research (18:3), 1981, pp. 382-388.

[17] M. Fornerino, A. Helme-Guizon, D. Gotteland, "Movie Consumption Experience and Immersion: Impact on Satisfaction", Recherche et Applications en Marketing (English Edition) (23:3), 2008, pp. 93-110.

[18] L.J. Francis, and J.E. Greer, "Measuring Attitude Towards Science Among Secondary School Students: The Affective Domain", Research in Science \& Technological Education (17:2), 1999, pp. 219-226.

[19] S. Geisser, "A Predictive Approach to the Random Effect Model”, Biometrika (61:1), 1974, pp. 101-107.

[20] D. Goodhue, W. Lewis, and R. Thompson, "Statistical Power in Analyzing Interaction Effects: Questioning the Advantage of PLS with Product Indicators", Information Systems Research (18:2), 2007, pp. 211-227.

[21] Hair, J. F., W.C. Black, B.J. Babin, and R.E. Anderson, Multivariate Data Analysis, Prentice Hall, Upper Saddle River, NJ, 2009.

[22] Hair, J.F., G.T.M. Hult, C.M. Ringle, and M. Sarstedt, A Primer on Partial Least Squares Structural Equation Modeling (PLS-SEM), Sage Publications, Thousand Oaks, CA, 2014. 
[23] E. Hartl, and B. Berger, "Escaping Reality: Examining the Role of Presence and Escapism in User Adoption of Virtual Reality Glasses", Proceedings of the 25th European Conference on Information Systems (ECIS), 2017, Guimarães, Portugal.

[24] C.-L. Hsu, "Exploring the Player Flow Experience in EGame Playing", International Journal of Technology and Human Interaction (6:2), 2010, pp. 47-64.

[25] C.-L. Hsu, and H.-P. Lu, "Why Do People Play On-Line Games? An Extended TAM with Social Influences and Flow Experience", Information \& Management (41:7), 2004, pp. 853-868.

[26] G.J. Johnson, G.C. Bruner II, and A. Kumar, "Interactivity and its Facets Revisited: Theory and Empirical Test”, Journal of Advertising (35:4), 2006, pp. 35-52.

[27] S.E. Kober, and C. Neuper, "Personality and Presence in Virtual Reality: Does Their Relationship Depend on the Used Presence Measure?", International Journal of HumanComputer Interaction (29:1), 2013, pp. 13-25.

[28] M.K. Lindell, and D.J. Whitney, "Accounting for Common Method Variance in Cross-Sectional Research Designs", Journal of Applied Psychology (86:1), 2001, pp. 114-121.

[29] Lohmöller, J.B., Latent Variable Path Modeling with Partial Least Squares, Physica, Heidelberg, Germany, 1989.

[30] P.B. Lowry, B. Hammer, J.E. Gaskin, T. Roberts, and N.W. Twyman, "Taking 'Fun and Games' Seriously: Proposing the Hedonic-Motivation System Adoption Model (HMSAM)", Journal of the Association for Information Systems (14:11), 2013, pp. 617-671.

[31] S.T. Lulu, "Place Presence, Social Presence, CoPresence, and Satisfaction in Virtual Worlds", Computers \& Education (58:1), 2012, pp. 154-161.

[32] M. Matsunaga, "How to Factor-Analyze Your Data Right: Do's, Don'ts, and How-To's", International Journal of Psychological Research (3:1), 2015, pp. 97-110.

[33] A. McMahan, "Immersion, Engagement and Presence: A Method for Analyzing 3D Video Games", in Wolf, M.J.P. and B. Perron (eds.), Video Game Theory Reader, Routledge, New York, NY, 2003, pp. 67-86.

[34] S.J. McMillan, and J.S. Hwang, "Measures of Perceived Interactivity: An Exploration of the Role of Direction of Communication, User Control, and Time in Shaping Perceptions of Interactivity", Journal of Advertising (31:3), 2002, pp. 29-42.

[35] J. Mütterlein, and T. Hess, "Exploring the Impacts of Virtual Reality on Business Models: The Case of the Media Industry", Proceedings of the 25th European Conference on Information Systems (ECIS), 2017, Guimarães, Portugal.
[36] J. Mütterlein, and T. Hess, "Immersion, Presence, Interactivity: Towards a Joint Understanding of Factors Influencing Virtual Reality Acceptance and Use", Proceedings of the 23rd Americas Conference on Information Systems (AMCIS), 2017, Boston, USA.

[37] F.F.-H. Nah, B. Eschenbrenner, and D. DeWester, "Enhancing Brand Equity through Flow and Telepresence: A Comparison of 2D and 3D Virtual Worlds", MIS Quarterly (35:3), 2011, pp. 731-747.

[38] F.F.-H. Nah, B. Eschenbrenner, Q. Zeng, V.R. Telaprolu, and S. Sepehr, "Flow in Gaming: Literature Synthesis and Framework Development", International Journal of Information Systems and Management (1:1-2), 2014, pp. 83-124.

[39] M.R. Nelson, R.A. Yaros, and H. Keum, "Examining the Influence of Telepresence on Spectator and Player Processing of Real and Fictitious Brands in a Computer Game", Journal of Advertising (35:4), 2006, pp. 87-99.

[40] R.L. Oliver, "A Cognitive Model of the Antecedents and Consequences of Satisfaction Decisions", Journal of Marketing Research (17:4), 1980, pp. 460-469.

[41] A. O'Cass, and J. Carlson, "Examining the Effects of Website-Induced Flow in Professional Sporting Team Websites", Internet Research (20:2), 2010, pp. 115-134.

[42] Rheinberg, F., Flow Short Scale, University of Potsdam, Potsdam, last retrieved $15^{\text {th }}$ of June 2017 , http://www.psych.uni-potsdam.de/people/rheinberg/messverf ahren/fks1-e.html.

[43] Rheingold, H., Virtual Reality: The Revolutionary Technology of Computer-Generated Artificial Worlds - And How it Promises and Threatens to Transform Business and Society, Summit Books/Simon and Schuster, New York, NY, 1991.

[44] Ringle, C. M., S. Wende, and J.-M. Becker, SmartPLS 3, SmartPLS GmbH, Bönningstedt, available at http://www.smartpls.com.

[45] B.O. Rothbaum, L. Hodges, S. Smith, J.H. Lee, and L. Price, "A Controlled Study of Virtual Reality Exposure Therapy for the Fear of Flying", Journal of Consulting and Clinical Psychology (68:6), 2000, pp. 1020-1026.

[46] Ryan, M.-L., Narrative as Virtual Reality 2: Revisiting Immersion and Interactivity in Literature and Electronic Media, John Hopkins University Press., Baltimore, MD, 2015.

[47] M. Slater, "Measuring Presence: A Response to the Witmer and Singer Presence Questionnaire", Presence: Teleoperators and Virtual Environments (8:5), 1999, pp. 560565.

[48] M. Slater, and S. Wilbur, "A Framework for Immersive Virtual Environments (FIVE): Speculations on the Role of 
Presence in Virtual Environments", Presence: Teleoperators and Virtual Environments (6:6), 1997, pp. 603-616.

[49] J. Steuer, "Defining Virtual Reality: Dimensions Determining Telepresence", Journal of Communication (42:4), 1992, pp. 73-93.

[50] M. Stone, "Cross-Validatory Choice and Assessment of Statistical Predictions", Journal of the Royal Statistical Society, Series B (Methodological) (36:2), 1974, pp. 111147.

[51] SuperData, and Unity, Can't Stop, Won't Stop: 2016 Mobile and VR Games in Review, SuperData Research and Unity Technologies, New York, NY and San Francisco, CA, 2017.

[52] I.E. Sutherland, "The Ultimate Display", Proceedings of the International Federation of Information Processing Congress Vol. 2, 1965, New York, NY.
[53] K.R. Walsh, and S.D. Pawlowski, "Virtual Reality: A Technology in Need of IS Research", Communications of the Association for Information Systems (8:article 20), 2002, pp. 297-313.

[54] B.G. Witmer, C.J. Jerome, and M.J. Singer, "The Factor Structure of the Presence Questionnaire", Presence: Teleoperators and Virtual Environments (14:3), 2005, pp 298-312.

[55] B.G. Witmer, and M.J. Singer, "Measuring Presence in Virtual Environments: A Presence Questionnaire", Presence: Teleoperators and Virtual Environments (7:3), 1998, pp. 225240. 